\title{
MicroRNA-16 inhibits the proliferation, migration and invasion of glioma cells by targeting Sal-like protein 4
}

\author{
YU ZHOU $^{1}$, YANG LIU ${ }^{2}, \mathrm{CHAO} \mathrm{HU}^{3}$ and YUGANG JIANG ${ }^{1}$ \\ Departments of ${ }^{1}$ Neurosurgery, ${ }^{2}$ Rehabilitation and ${ }^{3}$ Radiology, The Second Xiangya Hospital of \\ Central South University, Changsha, Hunan 410008, P.R. China
}

Received December 16, 2015; Accepted September 26, 2016

DOI: $10.3892 /$ ijmm.2016.2775

\begin{abstract}
MicroRNAs (miRNAs or miRs), a class of noncoding RNAs 18-25 nucleotides in length, act as key regulators in the development and malignant progression of various human cancers by modulating the expression of their target genes. Recently, miR-16 has been demonstrated to be play a role in glioma. However, the regulatory mechanisms of miR-16 in glioma growth and metastasis remain largely unclear. In the present study, qRT-PCR revealed that miR-16 was significantly downregulated in 23 glioma tissue specimens compared to 7 normal brain tissue specimens. Moreover, its levels were markedly lower in the glioma samples at stages T2-T4 compared to those at stage T1. The overexpression of miR-16 significantly suppressed the proliferation, migration and invasion of U251 and U87 glioma cells. Luciferase reporter assay identified Sal-like protein 4 (SALL4) as a target gene of miR-16, and its protein levels were found to be decreased in miR-16-overexpressing U251 and U87 cells. Furthermore, the overexpression of SALL4 significantly reversed the suppressive effects of miR-16 on the proliferation, migration and invasion of U251 and U87 cells, suggesting that miR-16 playsa tumor suppressor role in glioma by inhibiting cell proliferation and invasion through the targeting of SALL4. Finally, we found that SALL4 was significantly upregulated in glioma tissues compared to normal brain tissues, and its levels were markedly higher in the glioma tissues at stages T2-T4 compared to those at stage T1. In addition, the expression levels of SALL4 inversely correlated with the miR-16 levels in glioma tissues, suggesting that the downregulation of miR-16 contributes to the upregulation of SALL4 in glioma. On the whole, the findings of this study indicate a role for the miR-16/SALL4 axis in glioma. Our data may also provide a potential therapeutic target for the treatment of glioma.
\end{abstract}

Correspondence to: Professor Yugang Jiang, Department of Neurosurgery, The Second Xiangya Hospital of Central South University, 139 Renmin Road, Changsha, Hunan 410008, P.R. China E-mail: jiangyg01@sina.com

Key words: glioma, microRNA-16, Sal-like protein 4, proliferation, migration, invasion

\section{Instruction}

Glioma is the most common malignant tumor of the brain, and accounts for approximately $30 \%$ of central nervous system tumors and $80 \%$ of all malignant brain tumors (1). Despite the remarkable development of therapies for other types of cancer in recent decades, the prognosis of patients with advanced glioma remains poor, mainly due to its resistance to radiotherapy, chemotherapy and adjuvant therapies (2-5). The deregulation of oncogenes or tumor suppressors has been implicated in glioma (6). Therefore, the investigation of the roles of genetic and epigenetic factors may aid in the developmetn of novel diagnostic and therapeutic strategies for glioma (7).

MicroRNAs (miRNAs or miRs), a class of non-coding RNAs 18-25 nucleotides in length, which are able to suppress gene expression by targeting the complementary regions of mRNAs and inhibiting protein translation (8). By negatively mediating their target genes, miRNAs act as key regulators in a variety of physiological and pathological biological processes, including tumorigenesis $(9,10)$. The deregulation of miRNAs has been observed in glioma, and is associated with tumor growth, metastasis and drug resistance $(11,12)$. Therefore, the investigation of the regulatory mechanisms of miRNAs is important for the treatment of glioma.

Recently, miR-16 has been implicated in the development and progression of glioma (13). Malzkorn et al investigated the expression profiles of 157 miRNAs in 4 patients with primary WHO grade II gliomas that spontaneously progressed to WHO grade IV secondary glioblastomas, and found that miR-16 showed increased an expression upon progression, suggesting that its upregulation may play a role in the progression of glioma (14). On the contrary, however, the following studies identified miR-16 as a tumor suppressor in glioma, by demonstrating that miR-16 exerts inhibitory effects on growth, migration, invasion, epithelial-mesenchymal transition (EMT), and angiogenesis in glioma (13,15-17). Moreover, several targets of miR-16 have been identified in glioma, including Zyxin, Bcl-2, matrix metalloproteinase (MMP)9 and BMI1 proto-oncogene, polycomb ring finger Bmi-1 $(13,15,17)$. As one miRNA has many targets (18), whether other targets are also involved in the miR-16-mediated inhibition of glioma remains unknown.

Sal-like protein 4 (SALL4) is a zinc finger transcription factor, and has been identified as a marker for stem cells, involved in the maintenance of self-renewal in embryonic stem 
cells (19). Moreover, SALL4 was recently identified as an important biomarker for several common human cancers $(20,21)$. Zhang et al found that SALL4 was significantly upregulated in glioma, and a high level of SALL4 expression correlated with a poor outcome (22). A previous study demonstrated that the upregulation of SALL4, caused by the low expression of miR-107, inhibited cell apoptosis in glioma (23). Accordingly, SALL4 acts as an oncogene in glioma, and may become an important target for the treatment of glioma. However, evidence of the regulatory mechanisms of SALL4 expression in glioma is limited.

In the present study, we aimed to investigate the regulatory mechanisms of miR-16 in glioma growth and metastasis. We found that miR-16 was significantly downregulated in glioma tissues compared to normal brain tissues, and that the reduced miR-16 levels were associated with a greater malignancy of glioma. We further revealed that miR-16 inhibited cell proliferation, migration and invasion, and EMT in glioma, at least in part by directly targeting SALL4, which was markedly upregulated in glioma tissues and inversely correlated with the miR-16 levels.

\section{Materials and methods}

Ethics statement and clinical sample collection. This study was approved by the Ethics Committee of Central South University, Changsha, China. A total of 23 cases of glioma specimens and 7 cases of non-tumorous brain tissues were collected from the Second Xiangya Hospital of Central South University between January 2012 and March 2013. Non-tumorous brain tissues were collected by the partial resection of normal brain tissue in order to reduce increased intracranial pressure in the treatment of severe head injury. All tissue samples were immediately snap-frozen in liquid nitrogen and stored at $-80^{\circ} \mathrm{C}$ until use.

Written informed consent was obtained from all patients involved in this study. All glioma patients included 14 males and 9 females who ranged in age from 35 to 71 years, with a mean age of 51.3 years. None of the patients had any radiotherapy or chemotherapy prior to surgical resection. The glioma specimens were classified according to the World Health Organization (WHO) criteria (24). Among these glioma samples, 5 cases were pilocytic astrocytomas (WHO I), 6 were diffuse astrocytomas (WHO II), 6 were anaplastic astrocytomas (WHO III), and 6 were glioblastomas (WHO IV).

Cell culture and transfection. The human glioma cell lines, U87 and U251, were obtained from the Chinese Academy of Sciences (Shanghai, China). The cells were cultured in Dulbecco's modified Eagle's medium (DMEM) supplemented with $10 \%$ fetal bovine serum (FBS) (both from Life Technologies, Carlsbad, CA, USA) at $37^{\circ} \mathrm{C}$ in a humidified incubator containing 5\% $\mathrm{CO}_{2}$. Lipofectamine 2000 (Life Technologies) was used to perform transfection according to the manufacturer's instructions. Briefly, the U87 and U251 cells were cultured to $70 \%$ confluence, and resuspended in serum-free medium. Scramble miR (miR-NC), miR-16 mimic, miR-16 inhibitor (all from Genecopoeia, Rockville, MD, USA), the pc-DNA3.1-SALL4 plasmid (Amspring, Changsha, China), and Lipofectamine 2000 were diluted in OPTI-MEM (Life Technologies). The diluted Lipofectamine 2000 was added to the diluted miR or plasmid, and incubated for $20 \mathrm{~min}$ at room temperature, and then added to the cell suspension. Following incubation at $37^{\circ} \mathrm{C}, 5 \% \mathrm{CO}_{2}$ for $6 \mathrm{~h}$, the transfection mixture was replaced with DMEM with $10 \%$ FBS. The cells were then cultured for $48 \mathrm{~h}$ prior to being used in the following assays.

Reverse transcription-quantitative PCR (RT-qPCR). Total RNA was extracted from the tissues and cells using TRIzol Reagent (Life Technologies) in accordance with the manufacturer' $s$ instructions. RT-qPCR was used to examine the relative miR-16 expression using the mirVana ${ }^{\mathrm{TM}}$ qRT-PCR microRNA detection kit (Life Technologies) in accordance with the manufacturer' $s$ instructions. U6 was used as an internal reference. The relative mRNA expression of SALL4 was detected by RT-qPCR using the standard SYBR-Green RT-PCR kit (Takara, Otsu, Japan) in accordance with the manufacturer's instructions. Glyceraldehyde 3-phosphate dehydrogenase (GAPDH) was used as an internal reference. For both miRNA and mRNA detection, the reaction conditions were $95^{\circ} \mathrm{C}$ for $3 \mathrm{~min}$, followed by 40 cycles of $95^{\circ} \mathrm{C}$ for $15 \mathrm{sec}$ and $60^{\circ} \mathrm{C}$ for $30 \mathrm{sec}$. The specific primers for miR-16 and U6 were purchased from Genecopoeia. The specific primers for SALL4 were as follows: forward, 5'-TAGCCCTGCGTA GCCAGTTA-3' and reverse, 5'-TCATGCTTAGTCCACT GTCTGT-3'. The specific primers for GAPDH were as follows: forward, 5'-ACAACTTTGGTATCGTGGAAGG-3' and reverse, 5'-GCCATCACGCCACAGTTTC-3'. The relative expression level was quantified using the $2^{-\Delta \Delta \mathrm{Ct}}$ method.

3-(4,5-Dimethylthiazol-2-yl)-2,5-diphenyltetrazolium bromide (MTT) assay. Cell proliferation was examined by MTT assay. The U87 and U251 cells $\left(2 \times 10^{3}\right)$ were seeded in a 96-well plate. Each well was supplemented with $100 \mu \mathrm{l}$ of fresh serum-free medium with $0.5 \mathrm{~g} / 1 \mathrm{MTT}$. Following incubation at $37^{\circ} \mathrm{C}, 5 \%$ $\mathrm{CO}_{2}$ for $12,24,48$ and $72 \mathrm{~h}$, the medium containing MTT was removed, and $50 \mu \mathrm{l}$ of DMSO were added to each well. Following incubation at $37^{\circ} \mathrm{C}, 5 \% \mathrm{CO}_{2}$ for $10 \mathrm{~min}$, the absorbance at $570 \mathrm{~nm}$ (A570) of each sample was measured using a plate reader (Tecan Infinite M200; Tecan, Männedorf, Switzerland).

Wound healing assay. Wound healing assay was used to examine the migratory capacity of the glioma cells. The U87 and U251 cells were cultured to full confluence. Wounds of approximately $1 \mathrm{~mm}$ in width were created using a plastic scriber. The cells were washed and then cultured in DMEM containing $10 \%$ FBS for $48 \mathrm{~h}$. The cells were then observed and photographed under a microscope (BX53; Olympus, Tokyo, Japan).

Transwell assay. Cell invasion was examined using 24-well Transwell chambers with a layer of Matrigel (Chemicon, Temecula, CA, USA). For each group, $300 \mu \mathrm{l}$ of cell suspension, each containing 5,000 cells, was added to the upper chamber. DMEM containing $10 \%$ FBS was added to the lower chamber. Following culture for $24 \mathrm{~h}$, non-invading cells on the interior of the inserts were removed using a cotton-tipped swab. Invading cells on the lower surface of the inserts were stained with $0.1 \%$ gentian violet (Sigma, St. Louis, MO, USA), rinsed with water, and dried in air. The invading cells were observed under a microscope (BX53; Olympus). The cell number was counted.

Western blot analysis. The cells were lysed in protein lysis buffer (Xinyu Biotechnology, Shanghai, China). The protein 

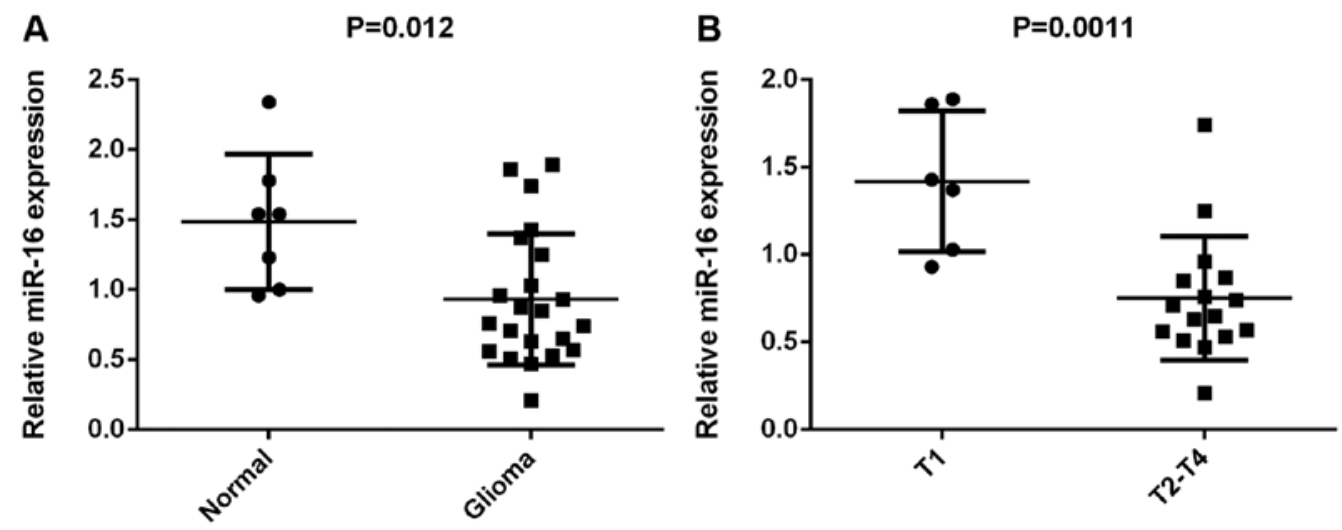

Figure 1. (A) RT-qPCR was used to determine the relative expression of miR-16 in 23 cases of glioma tissues and 7 cases of normal brain tissues (normal). (B) RT-qPCR was used to determine the relative expression of miR-16 in 17 cases of T2-T4 stage of glioma tissues and 6 cases of T1 stage of glioma tissues.

concentration was determined using the BCA Protein assay kit (Pierce Chemical Co., Rockford, IL, USA). Protein was separated with $10 \%$ sodium dodecyl sulfate-polyacrylamide gel electrophoresis (SDS-PAGE), transferred onto a PVDF membrane (Life Technologies), and then blocked in 5\% non-fat dried milk (Mengniu, Beijing, China) in TBST (Sigma) for $2 \mathrm{~h}$. The PVDF membrane was then incubated with primary antibodies against SALL4 (rabbit polyclonal; ab29112) or GAPDH (rabbit polyclonal; ab9485) (Abcam, Cambridge, MA, USA) at $4^{\circ} \mathrm{C}$ overnight, and then washed with TBST 4 times. The PVDF membrane was incubated with mouse anti-rabbit secondary antibody (ab99702; Abcam) for $1 \mathrm{~h}$ at room temperature, and then washed with TBST 3 times. The immune complexes were then detected using the ECL western blotting kit (Pierce Chemical Co.) and X-film (Kodak, Tokyo, Japan). ImageJ software was used to analyze the relative protein expression, represented as the density ratio versus GAPDH.

Bioinformatics analysis. TargetScan Human 7.0 online software (www.targetscan.org) was used to predict the putative target of miR-16.

Dual luciferase reporter assay. The wild-type (WT) sequence of the 3'UTR of SALL4 was constructed by PCR and inserted into the pMiR-Report miRNA Expression Reporter vector (Thermo Fisher Scientific, Carlsbad, CA USA). The mutant type (MT) sequence of the 3'UTR of SALL4 was constructed using the Easy Mutagenesis System kit (Promega, Madison, WI, USA) in accordance with the manufacturer's instructions, and then inserted into the pMiR-Report miRNA Expression Reporter vector. The U87 and U251 cells were co-transfected with the WT SALL4-3'UTR plasmid (200 ng) or the MT SALL4-3'UTR plasmid (200 ng), and miR-NC (100 nM) or miR-16 mimic (100 nM), using Lipofectamine 2000. Following co-transfection for $48 \mathrm{~h}$, the dual-luciferase reporter assay system (Promega) was used to determine the activities of Renilla luciferase and Firefly luciferase. The Renilla luciferase activity was normalized to the Firefly luciferase activity.

Statistical analysis. The data in this study are expressed as the means \pm SD. Statistical analysis was performed using SPSS 17.0 (SPSS, Armonk, NY, USA). The differences between 2 groups were analyzed using the Student' t-test. The differences among more than 2 groups were analyzed using ANOVA. A value of $\mathrm{P}<0.05$ was considered to indicate a statistically significant difference.

\section{Results}

miR-16 is downregulated in glioma. To reveal the role of miR-16 in glioma, RT-qPCR was used to determine its expression levels. The expression levels of miR-16 were markedly reduced in the glioma tissues compared to the normal brain tissues (Fig. 1). Moreover, its levels were markedly lower in the glioma tissues at stages T2-T4 compared to those at stage T1, suggesting that its downregulation was associated with the malignant progression of glioma. Therefore, miR-16 is downregulated in glioma, and the lower miR-16 levels were associated with its malignant progression.

miR-16 inhibits the proliferation, migration and invasion of U87 and U251 glioma cells. As miR-16 was found to be downregulated in glioma, we transfected the U87 and U251 glioma cells with miR-16 mimic in order to upregulate its expression. Following transfection, the miR-16 levels were markedly increased compared with those in the cells transfected with the scramble (control) miR (Fig. 2A and B). MTT assay, wound healing assay and Transwell assay were further used to examine the proliferation, migration and invasion of glioma cells, respectively. We observed that the overexpression of miR-16 significantly suppressed the proliferation, migration and invasion of the U87 and U251 cells compared to the control cells (Fig. 2C-H). Therefore, miR-16 exerts inhibitory effects on the proliferation, migration and invasion of glioma cells.

SALL4, a target gene of miR-16, is negatively mediated by miR-16 in glioma cells. We then investigated the putative targets of miR-16 in glioma cells. Bioinformatics analysis predicted that SALL4 was a potential target gene of miR-16, and their targeting relationship was evolutionarily conserved (Fig. 3A and B). To verify their targeting relationship, the luciferase reporter plamids containing the WT or MT of SALL4 3'UTR were generated (Fig. 3C and D), and luciferase reporter assay was conducted using the U87 and U251 cells. As demonstrated in Fig. 3E and F, 

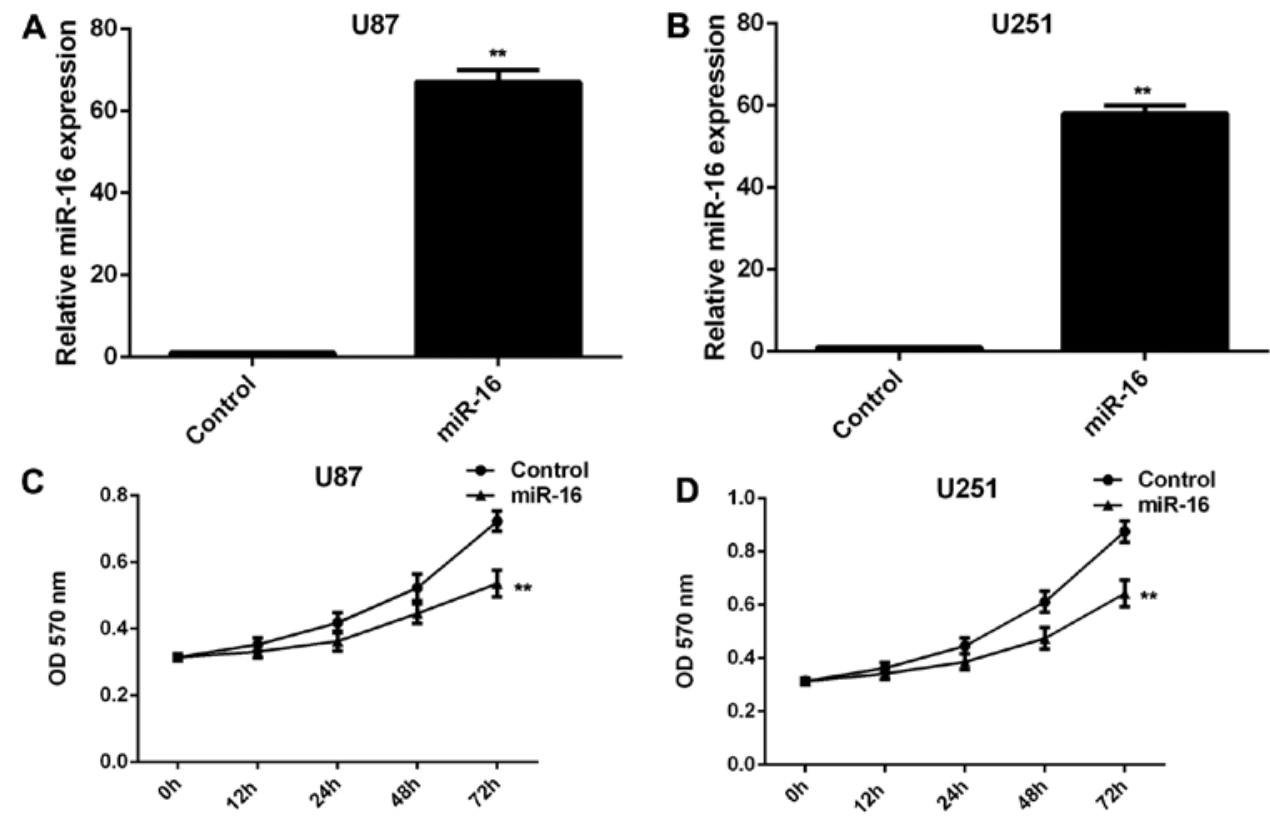

$\mathbf{E}$

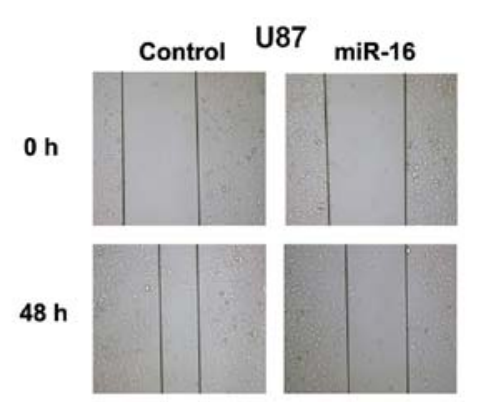

$\mathbf{F}$
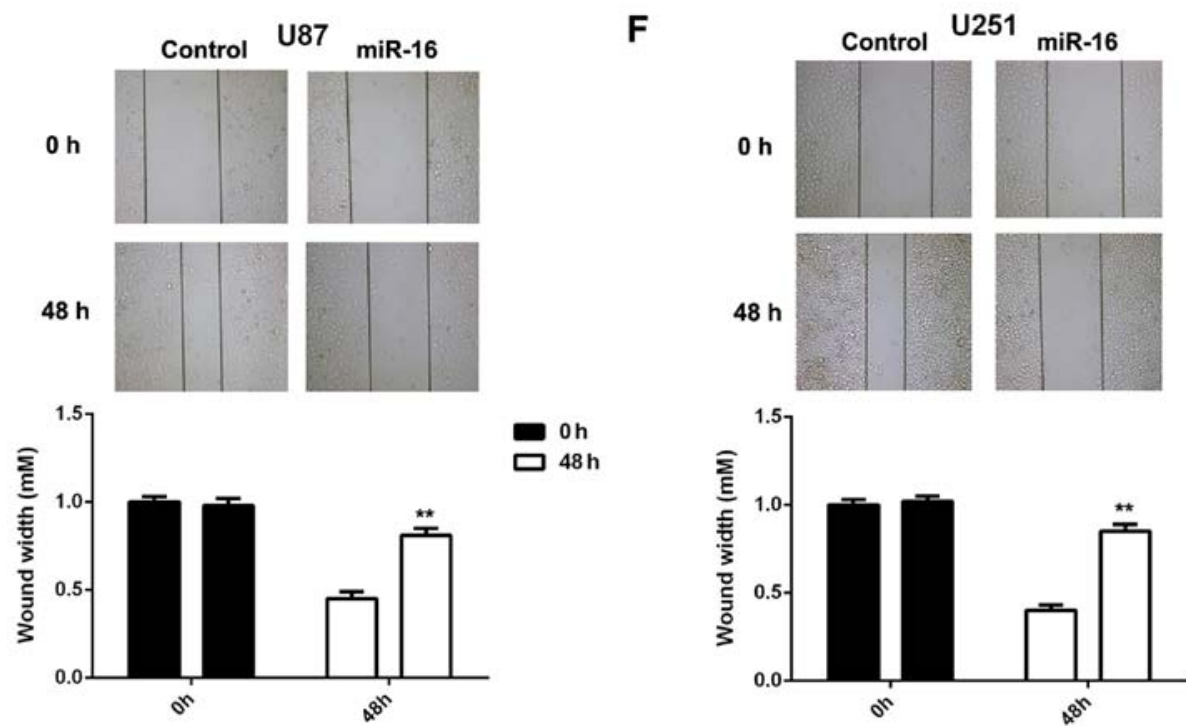

G

U87

miR-16
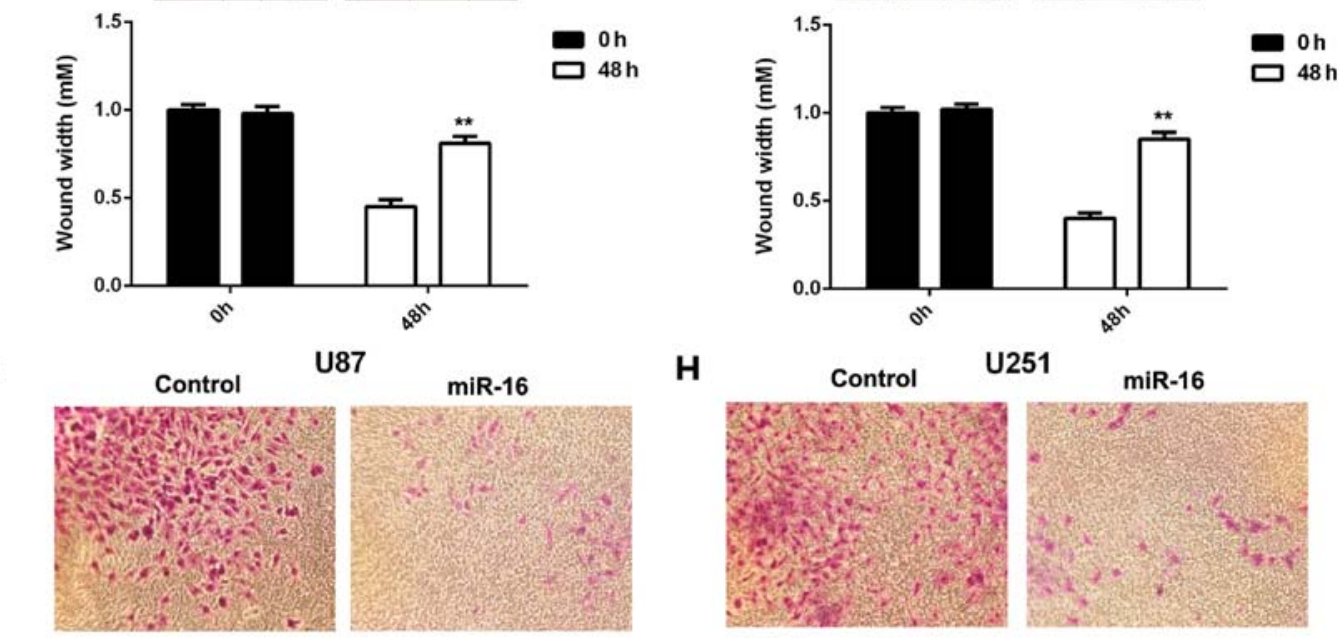

H
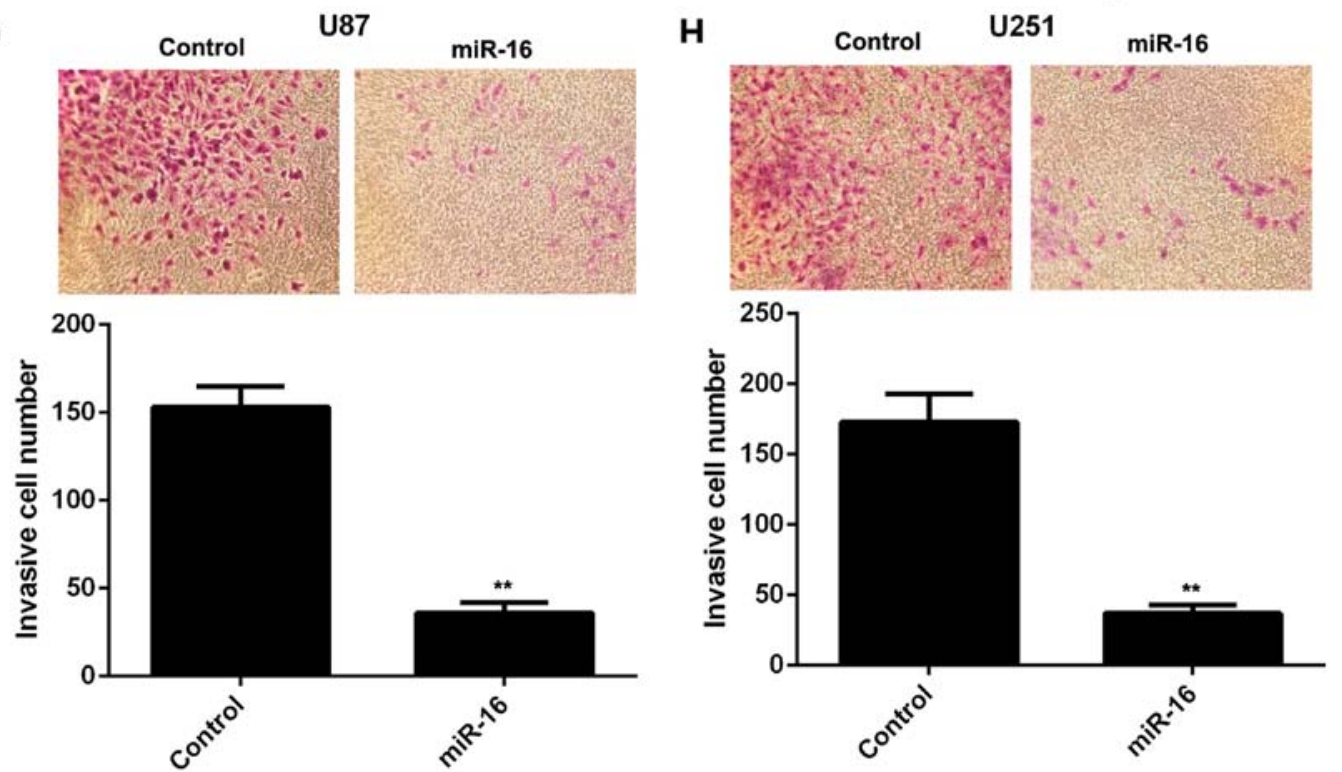

Figure 2. (A and B) RT-qPCR was performed to determine the relative expression of miR-16 in U87 and U251 cells transfected with scramble miR (control) or miR-16 mimic. (C and D) MTT assay, (E and F) wound healing assay, and (G and H) Transwell assay were used to determine cell proliferation, migration and invasion in each group, respectively. ${ }^{* *} \mathrm{P}<0.01$ vs. control (scramble). 
A

\begin{tabular}{|c|c|c|c|c|c|c|c|}
\hline & $\begin{array}{l}\text { Predicted consequential pairing of target region (top) } \\
\text { and miRNA (bottom) }\end{array}$ & $\begin{array}{l}\text { Site } \\
\text { type }\end{array}$ & $\begin{array}{l}\text { Context++ } \\
\text { score }\end{array}$ & $\begin{array}{l}\text { Context++ score } \\
\text { percentile }\end{array}$ & $\begin{array}{l}\text { Weighted } \\
\text { context }++ \text { score }\end{array}$ & $\begin{array}{l}\text { Conserved branch } \\
\text { length }\end{array}$ & $\mathrm{P}_{\mathrm{CT}}$ \\
\hline $\begin{array}{l}\text { Position 175-181 of SALL4 } 3 \text { UTR } \\
\text { hsa-miR-16-5p }\end{array}$ & 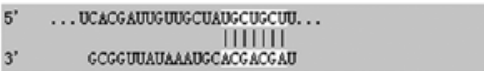 & $\begin{array}{l}7 \mathrm{mer}- \\
\mathrm{m} 8\end{array}$ & -0.27 & 93 & -0.27 & 3.888 & 0.61 \\
\hline
\end{tabular}

B

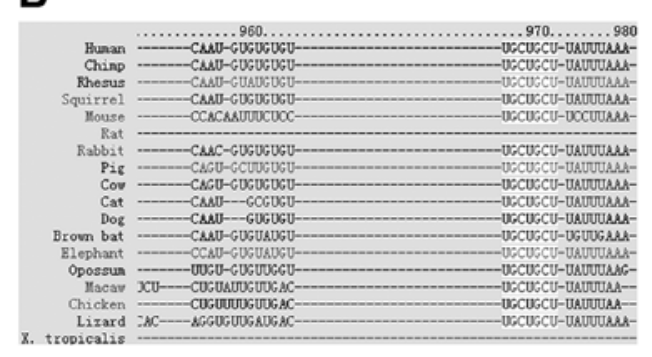

C

\author{
WT SALL4 3'UTR 5'...CACGAUUGUUGCUAUGCUGCUU....3' \\ || || || | \\ miR-16 3'...GCGGUUAUAAAUGCACGACGAU...5' \\ III \\ MT SALL4 3'UTR 5'...CACGAUUGUUGCUAUGCAAAUU...3'
}

D

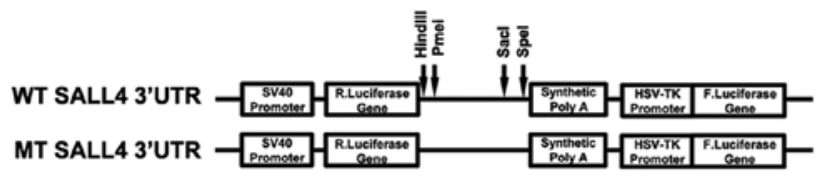

$\mathbf{F}$

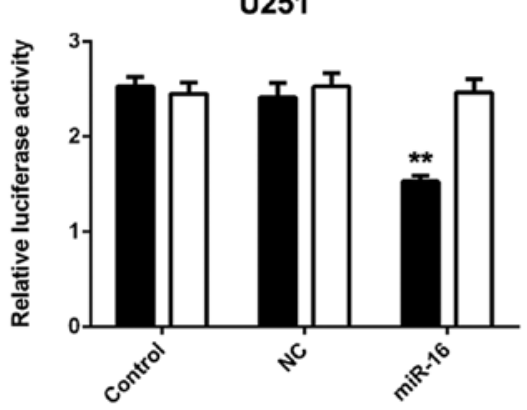

H

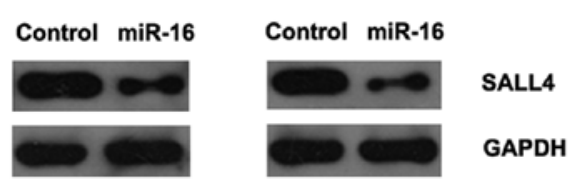

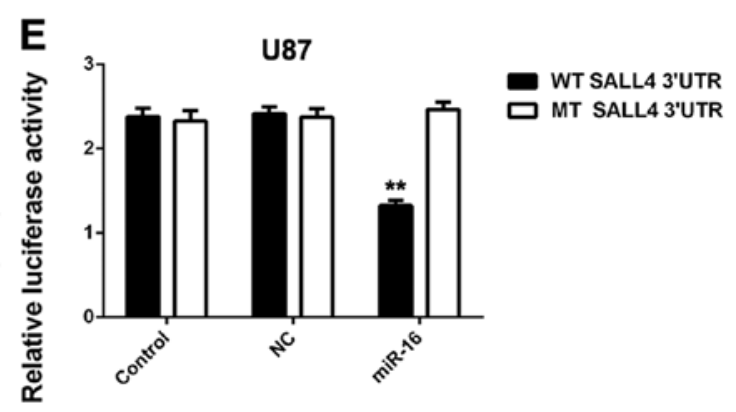
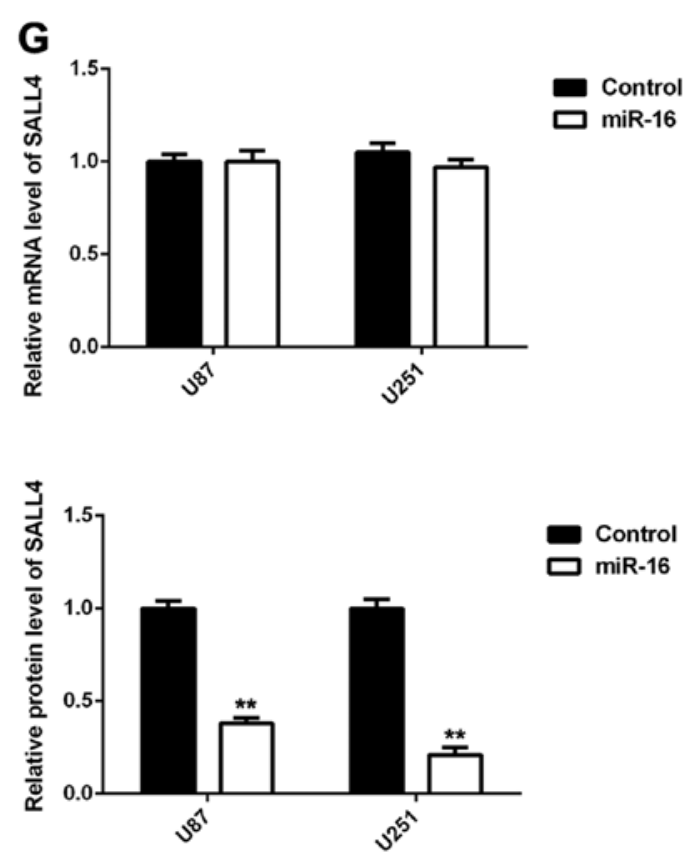

Figure 3. (A) Targetscan software predicted that Sal-like protein 4 (SALL4) was a potential target gene of miR-16, and (B) their targeting relationship was evolutionarily conserved. (C) The seed sequences of miR-16 in the wild-type (WT) or mutant type (MT) of SALL4 3'UTR are indicated. (D) The WT or MT SALL4 3'UTR were cloned into the luciferase reporter vector. (E and F) The luciferase activity was significantly decreased in U87 and U251 cells co-transfected with the WT SALL4 vector and miR-16 mimic, but was unaltered in U87 and U251 cells co-transfected with the MT SALL4 vector and miR-16 mimic, compared to the control group. Control: U87 and U251 cells only transfected with WT SALL4 or MT SALL4 vectors, respectively. NC: cells co-transfected with WT SALL4 or MT SALL4 vector, and scramble miR. ${ }^{* *} \mathrm{P}<0.01$ vs. control. (G) RT-qPCR and $(\mathrm{H})$ western blot analysis were used to examine the mRNA and protein levels, respectively of SALL4 in U87 and U251 cells transfected with scramble miR (control) or miR-16 mimic. ${ }^{* *} \mathrm{P}<0.01$ vs. control.

the luciferase activity was significantly decreased in the U87 and U251 cells co-transfected with the WT SALL4 3'UTR plamid and miR-16 mimic, but was unaltered in the U87 and U251 cells co-transfected with the MT SALL4 3'UTR plamid and miR-16 mimic, when compared to the control group, respectively, indicating that SALL4 is a direct target gene of miR-16.
As miRNAs inhibit the expression of their target genes, we then examined the effects of miR-16 overexpression on the expression of SALL4 in glioma cells. We found that the overexpression of miR-16 did not affect the mRNA expression of SALL4 (Fig. 3G), but significantly inhibited the protein expression of SALL4 in the U87 and U251 cells (Fig. 3H). 
A
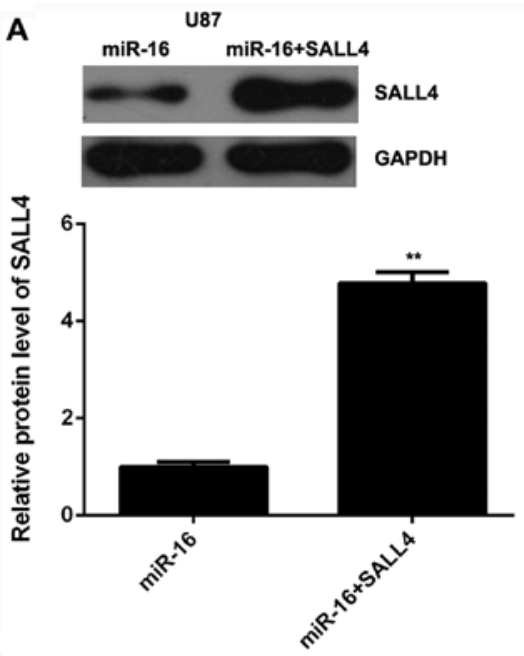

$$
\text { C }
$$

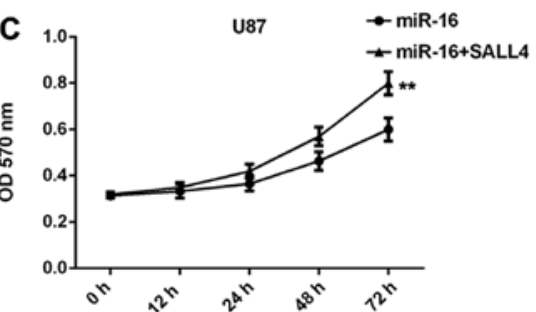

E

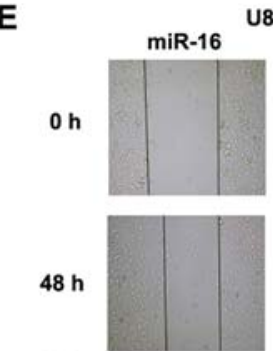

U87

miR-16+SALL4
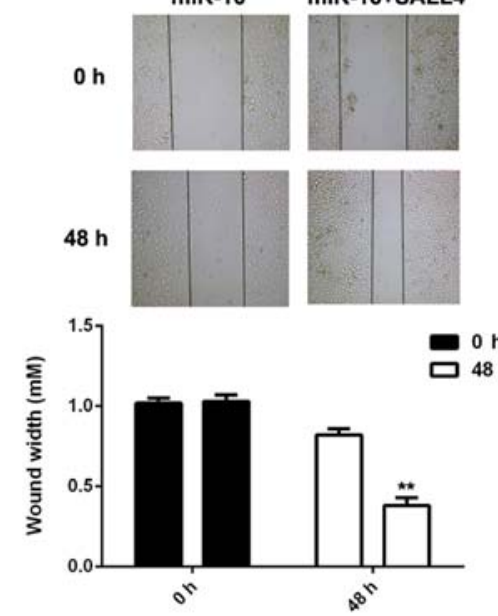

G
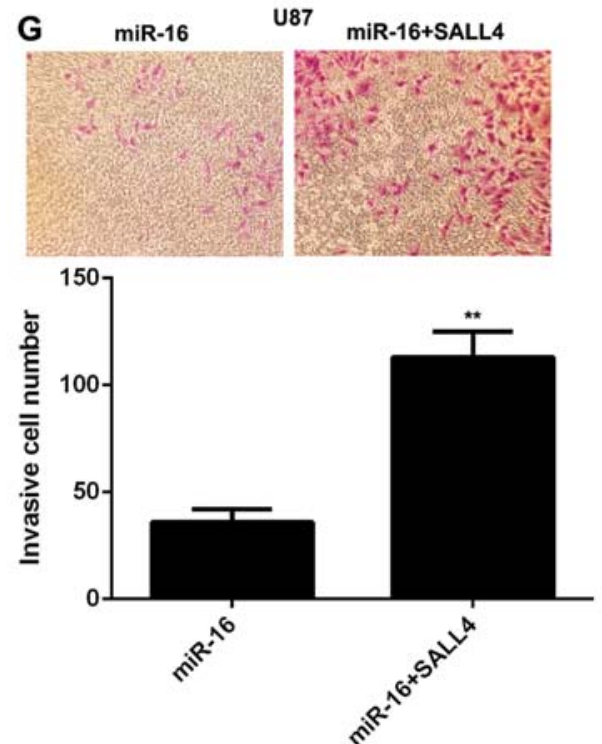
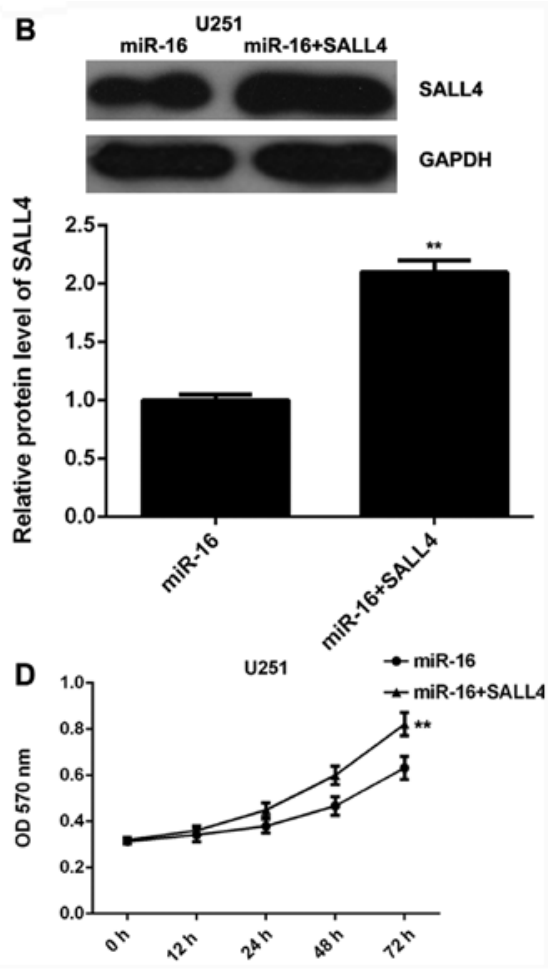

F
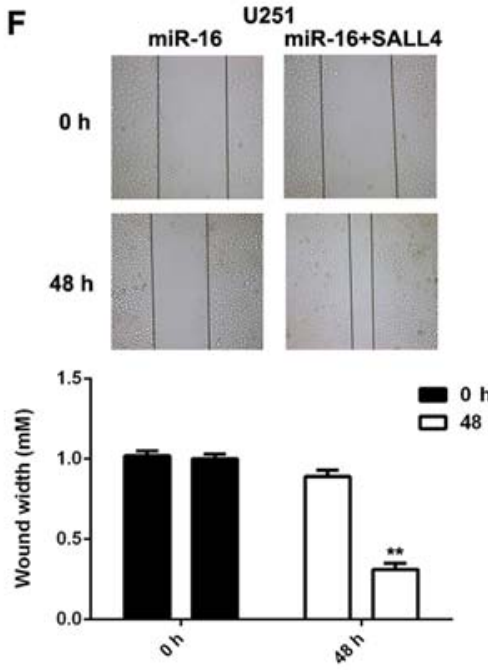

H
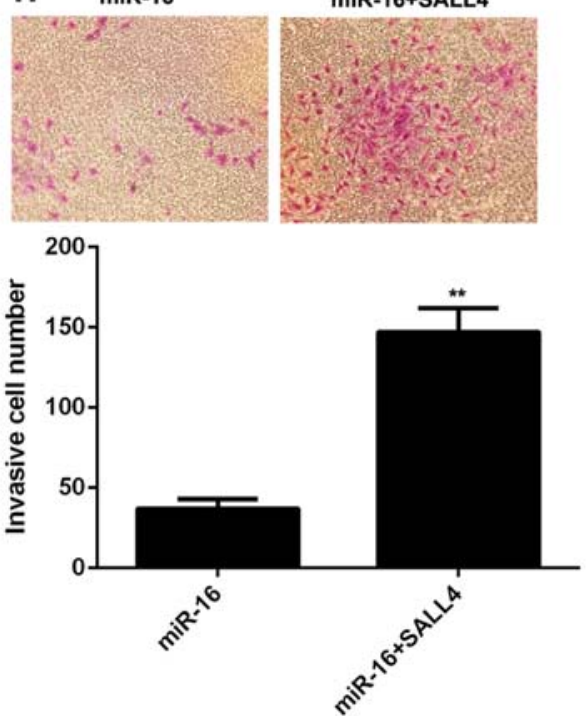

Figure 4. U87 and U251 cells were transfected with miR-16 mimic with or without pc-DNA3.1-Sal-like protein 4 (SALL4) plasmid. (A and B) Following transfection, the protein level of SALL4 was then examined by western blot analysis. (C and D) MTT assay, (E and F) wound healing assay, and (G and H) Transwell assay were conducted to examine cell proliferation, migration and invasion, respectively. $\mathrm{P}<0.05$ vs. transfection with miR-16 only. 

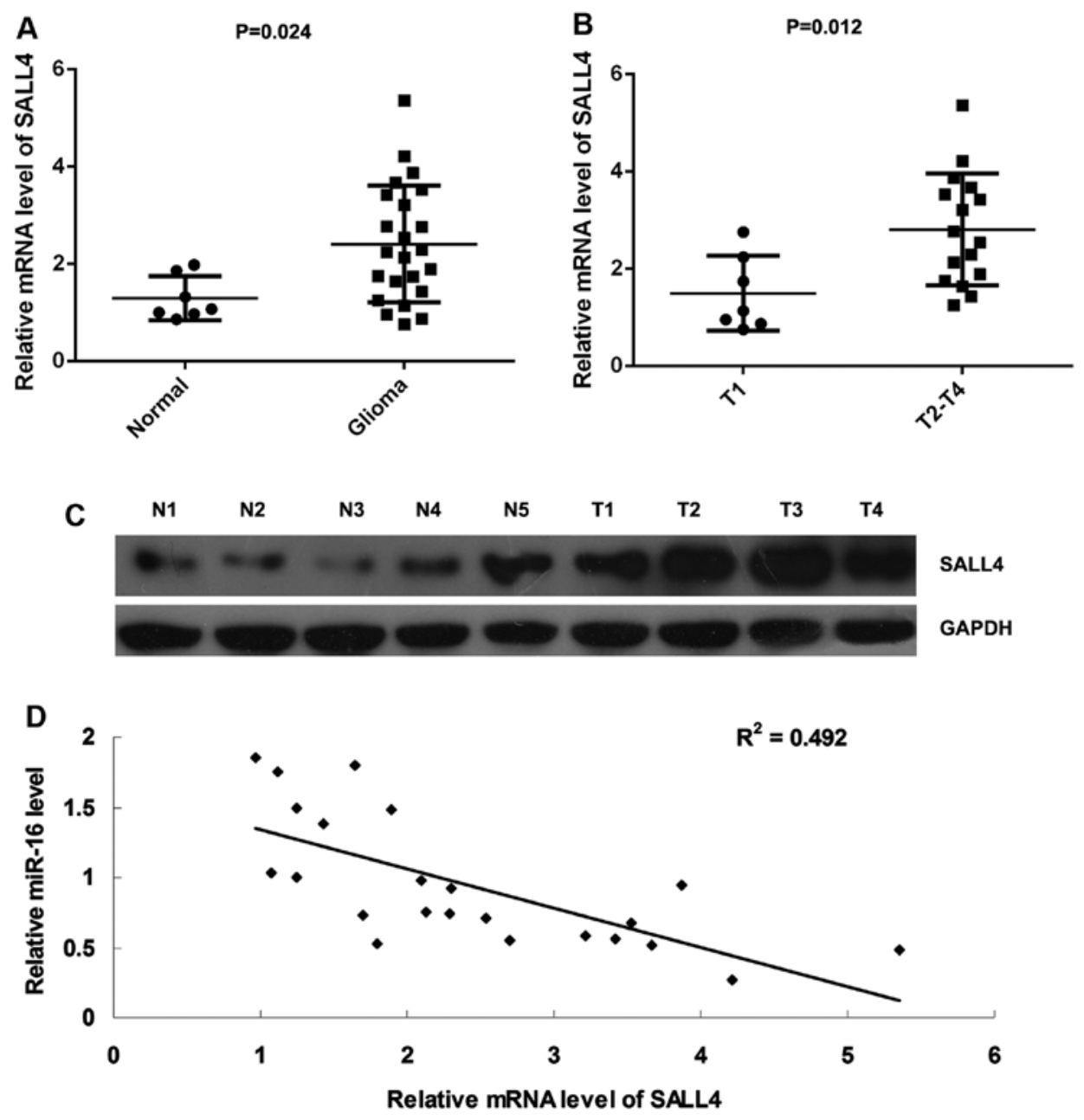

Figure 5. (A) RT-qPCR was used to determine the mRNA expression of Sal-like protein 4 (SALL4) in 23 cases of glioma tissues and 7 cases of normal brain tissues (normal). " $\mathrm{P}<0.01$ vs. normal tissues. (B) RT-qPCR was used to determine the mRNA expression of SALL4 in 17 cases of T2-T4 stage of glioma tissues and 6 cases of $\mathrm{T} 1$ stage of glioma tissues. ${ }^{* *} \mathrm{P}<0.01 \mathrm{vs}$. T1. (C) Western blot analysis was used to determine the protein level of SALL4 in glioma tissues (T) and normal brain tissues (N). (D) The correlation between the expression levels of miR-16 and SALL4 was analyzed in 23 cases of glioma tissues.

Therefore, miR-16 can inhibit the expression of SALL4 at the post-transcriptional level in glioma cells by directly targeting the 3'UTR of SALL4 mRNA.

SALL4 is involved in the miR-16-mediated inhibition of the proliferation, migration and invasion of glioma cells. As SALL4 has been reported to be upregulated in glioma and to be associated with its malignant progression (22), we speculated that SALL4 may be involved in the miR-16-mediated proliferation, migration and invasion of glioma cells. miR-16-overexpressing glioma cells were transfected with the pc-DNA3.1-SALL4 plasmid to restore SALL4 expression. Following transfection, western blot analysis was conducted to examine the protein levels of SALL4 in each group. The SALL4 protein levels were markedly higher in the U87 and U251 cells co-trasnfected with the miR-16 mimic and SALL4 overexpression plasmid, when compared to the U87 and U251 cells transfected only with the miR-16 mimic (Fig. 4A and B). Subsequently, MTT assay, wound healing assay and Transwell assay were performed to examine the proliferation, migration and invasion of glioma cells in each group, respectively. Our data demonstrated that the proliferation, migration and invasion of thye U87 and U251 cells were significantly enhanced following co-transfection with themiR-16 mimic and SALL4 plasmid, when compared to the cells transfected only with the miR-16 mimic (Fig. 4C-H), indicating that the overexpression of SALL4 reversed the suppressive effects of miR-16 overexpression on glioma cell proliferation, migration and invasion. Based on these data, we suggest that miR-16 inhibits the proliferation, migration and invasion of glioma cells, at least partly by directly targeting SALL4.

SALL4 is upregulated in glioma tissues and its expression inversely correlates with the miR-16 levels. Finally, we conducted RT-qPCR to examine the mRNA levels of SALL4 in glioma tissues and normal brain tissues. We found that SALL4 was significantly upregulated in thye glioma tissues compared with the normal brain tissues (Fig. 5A). Moreover, its mRNA levels were markedly higher in the glioma samples at stages T2-T4, when compared with those at T1 stage, suggesting that its upregulation was associated with the malignant progression of glioma (Fig. 5B). Moreover, the results of western blot analysis further demonstrated that the protein expression of SALL4 was increased in the glioma tissues compared with the normal brain tissues (Fig. 5C). In addition, we found that the mRNA levels of SALL4 inversely correlated 
with the miR-16 levels in glioma tissues (Fig. 5D), suggesting that the downregulation of miR-16 may be an important cause for the upregulation of SALL4 in glioma.

\section{Discussion}

Recently, miR-16 has been demonstrated to play a role in the inhibition of the growth and metastasis of glioma $(13,15-17)$. However, the underlying mechanisms remain to be fully elucidated. In this study, we found that the miR-16 levels were markedly decreased in glioma tissues compared to normal brain tissues, and its downregulation was associated with the malignant progression of the disease. Further in vitro experiments revealed that the overexpression of miR-16 suppressed the proliferation, migration and invasion of glioma cells by directly targeting SALL4, which was significantly upregulated and inversely correlated with the miR-16 levels in glioma tissues.

The central tumor suppressor, p53, has been found to enhance the post-transcriptional maturation of miR-16 with growth-suppressive function in response to DNA damage (25). Moreover, miR-16 has been demonstrated to be deregulated and to play a role in a variety of human cancers. Amaral et al reported that miR-16 was significantly downregulated in ACTH-secreting pituitary tumors when compared to normal pituitary tissues (26). Furthermore, miR-16 has been shown to be deleted or downregulated in the majority of chronic lymphocytic leukemia cases, and to induce the apoptosis of leukemic cells by directly targeting Bcl-2 (27). Recently, miR-16 was found to act as a tumor suppressor in glioma (13,15-17). For instance, Li et al found that miR-16 inhibited the expression of Zyxin, and suppressed the proliferation, migration and invasion of high-invasive glioma cells (13). Wang et al reported that miR-16 suppressed the invasion, adhesion, cell cycle progression, production of interleukin (IL)-6, IL-8 and transforming growth factor- $\beta$, and EMT-related gene expression, including vimentin, $\beta$-catenin and E-cadherin in U87 and U251 glioma cells (16). Yang et al showed that miR-16 suppressed glioma cell growth and invasion, while it induced cell apoptosis in vitro and in vivo by inhibiting NF- $\mathrm{B} 1, \mathrm{MMP} 9$ and Bcl-2 (17). In the present study, we found that miR-16 was downregulated in glioma tissues compared to normal brain tissues, and its downregulation was associated with the tumor grade, TMN stage and vascular invasion of glioma. These data suggest that miR-16 may serve as a diagnostic marker for glioma.

Moreover, we identified SALL4 as a direct target gene of miR-16 by using luciferase reporter assay, and found that the overexpression of miR-16 led to a significant decrease in the protein levels of SALL4 in glioma U87 and U251 cells. SALL4, a stem cell-related transcription factor, has recently been demonstrated to play an oncogenic role in many common human cancers, such as hepatocellular carcinoma, endometrial cancer, lung cancer, colorectal cancer, esophageal squamous cell carcinoma and breast cancer, by enhancing tumor cell survival, growth, metastasis, angiogenesis and drug resistance (28-33). Recently, Zhang et al reported that the expression of SALL4 was significantly increased in glioma specimens compared to normal brain tissues, and that its upregulation tightly correlated with a higher pathological grade, as well as with a poor prognosis (22). Moreover, the knockdown of SALL4 effectively suppressed the proliferation of U251 cells (22). Accordingly, we hypothesized that the tumor suppressive role of miR-16 may be mediated through the inhibition of SALL4. To verify this hypothesis, miR-16-overexpressing glioma cells were further transfected with a pc-DNA3.1-SALL4 plasmid to restore its protein levels. Our data indicated that the overexpression of SALL4 reversed the inhibitory effects of miR-16 on the proliferation, migration and invasion of U87 and U251 cells. Therefore, we suggest that miR-16 inhibits the malignant phenotypes of glioma cells by directly targeting SALL4. We further found that SALL4 was upregulated in glioma, consistent with previous findings (22), and that its upregulation inversely correlated with the downregulation of miR-16 in glioma tissues. These data further suggest that downregulation of miR-16 may contribute to the upregulation of SALL4 in glioma. In future studies, we aim to further investigate the role of miR-16 and SALL4 in glioma in vivo, and to explore the downstream factors of the miR-16/SALL4 axis in glioma.

In addition, another miRNA (miR-107) was found to play a role in glioma by targeting SALL4. He et al reported that miR-107 was significantly downregulated in glioma, and suppressed glioma cell growth by directly targeting SALL4, leading to the activation of FADD/caspase-8/caspase-3/7 signaling pathway of cell apoptosis (23). Therefore, our study expands the understanding of the functions of miRNAs in glioma.

In conclusion, this study demonstrates that miR-16 plays a suppressive role in regulating cell proliferation, migration and invasion, and EMT in glioma, at least in part by directly targeting SALL4. Therefore, we suggest that the miR-16/ SALL4 axis may serve as a potential therapeutic target for the treatment of glioma.

\section{References}

1. Goodenberger ML and Jenkins RB: Genetics of adult glioma. Cancer Genet 205: 613-621, 2012.

2. Stewart LA: Chemotherapy in adult high-grade glioma: A systematic review and meta-analysis of individual patient data from 12 randomised trials. Lancet 359: 1011-1018, 2002.

3. Zhu VF, Yang J, Lebrun DG and Li M: Understanding the role of cytokines in Glioblastoma Multiforme pathogenesis. Cancer Lett 316: 139-150, 2012.

4. Sathornsumetee S, Reardon DA, Desjardins A, Quinn JA, Vredenburgh JJ and Rich JN: Molecularly targeted therapy for malignant glioma. Cancer 110: 13-24, 2007.

5. Pulkkanen KJ and Yla-Herttuala S: Gene therapy for malignant glioma: Current clinical status. Mol Ther 12: 585-598, 2005.

6. Marumoto T and Saya H: Molecular biology of glioma. Adv Exp Med Biol 746: 2-11, 2012.

7. Chen C and Wang G: Mechanisms of hepatocellular carcinoma and challenges and opportunities for molecular targeted therapy. World J Hepatol 7: 1964-1970, 2015.

8. Ambros V: The functions of animal microRNAs. Nature 431: 350-355, 2004.

9. Tessitore A, Cicciarelli G, Del Vecchio F, Gaggiano A, Verzella D, Fischietti M, Vecchiotti D, Capece D, Zazzeroni F and Alesse E: MicroRNAs in the DNA Damage/Repair Network and Cancer. Int J Genomics 2014: 820248, 2014.

10. Esquela-Kerscher A and Slack FJ: Oncomirs - microRNAs with a role in cancer. Nat Rev Cancer 6: 259-269, 2006.

11. Brower JV, Clark PA, Lyon W and Kuo JS: MicroRNAs in cancer: Glioblastoma and glioblastoma cancer stem cells. Neurochem Int 77: 68-77, 2014.

12. Auffinger B, Thaci B, Ahmed A, Ulasov I and Lesniak MS: MicroRNA targeting as a therapeutic strategy against glioma. Curr Mol Med 13: 535-542, 2013.

13. Li X, Ling N, Bai Y, Dong W, Hui GZ, Liu D, Zhao J and Hu J: MiR-16-1 plays a role in reducing migration and invasion of glioma cells. Anat Rec (Hoboken) 296: 427-432, 2013. 
14. Malzkorn B, Wolter M, Liesenberg F, Grzendowski M, Stühler K, Meyer HE and Reifenberger G: Identification and functional characterization of microRNAs involved in the malignant progression of gliomas. Brain Pathol 20: 539-550, 2010.

15. Chen F, Chen L, He H, Huang W, Zhang R, Li P, Meng Y and Jiang X: Up-regulation of microRNA-16 in glioblastoma inhibits the function of endothelial cells and tumor angiogenesis by targeting Bmi-1. Anticancer Agents Med Chem 16: 609-620, 2016.

16. Wang Q, Li X, Zhu Y and Yang P: MicroRNA-16 suppresses epithelial-mesenchymal transition related gene expression in human glioma. Mol Med Rep 10: 3310-3314, 2014.

17. Yang TQ, Lu XJ, Wu TF, Ding DD, Zhao ZH, Chen GL, Xie XS, Li B, Wei YX, Guo LC, et al: MicroRNA-16 inhibits glioma cell growth and invasion through suppression of $\mathrm{Bcl}-2$ and the nuclear factor- $\kappa \mathrm{B} 1 / \mathrm{MMP} 9$ signaling pathway. Cancer Sci 105: 265-271, 2014.

18. Ambros V: microRNAs: Tiny regulators with great potential. Cell 107: 823-826, 2001.

19. Chen X, Vega VB and $\mathrm{Ng} \mathrm{HH}$ : Transcriptional regulatory networks in embryonic stem cells. Cold Spring Harb Symp Quant Biol 73: 203-209, 2008.

20. Zhang X, Yuan X, Zhu W, Qian H and Xu W: SALL4: An emerging cancer biomarker and target. Cancer Lett 357: 55-62, 2015.

21. Oishi N, Yamashita T and Kaneko S: Molecular biology of liver cancer stem cells. Liver Cancer 3: 71-84, 2014.

22. Zhang L, Yan Y, Jiang Y, Cui Y, Zou Y, Qian J, Luo C, Lu Y and $\mathrm{Wu} X$ : The expression of SALL4 in patients with gliomas: High level of SALL4 expression is correlated with poor outcome. J Neurooncol 121: 261-268, 2015.

23. He J, Zhang W, Zhou Q, Zhao T, Song Y, Chai L and Li Y: Low-expression of microRNA-107 inhibits cell apoptosis in glioma by upregulation of SALL4. Int J Biochem Cell Biol 45: 1962-1973, 2013.

24. Cunliffe CH, Fischer I, Parag Y and Fowkes ME: State-of-the-art pathology: new WHO classification, implications, and new developments. Neuroimaging Clin N Am 20: 259-271, 2010.
25. Suzuki HI, Yamagata K, Sugimoto K, Iwamoto T, Kato S and Miyazono K: Modulation of microRNA processing by $\mathrm{p} 53$. Nature 460: 529-533, 2009

26. Amaral FC, Torres N, Saggioro F, Neder L, Machado HR, Silva WA Jr, Moreira AC and Castro M: MicroRNAs differentially expressed in ACTH-secreting pituitary tumors. J Clin Endocrinol Metab 94: 320-323, 2009.

27. Cimmino A, Calin GA, Fabbri M, Iorio MV, Ferracin M, Shimizu M, Wojcik SE, Aqeilan RI, Zupo S, Dono M, et al: miR-15 and miR-16 induce apoptosis by targeting Bcl-2. Proc Natl Acad Sci USA 102: 13944-13949, 2005.

28. Oikawa T, Kamiya A, Zeniya M, Chikada H, Hyuck AD, Yamazaki Y, Wauthier E, Tajiri H, Miller LD, Wang XW, et al: Sal-like protein 4 (SALL4), a stem cell biomarker in liver cancers. Hepatology 57: 1469-1483, 2013.

29. Li A, Jiao Y, Yong KJ, Wang F, Gao C, Yan B, Srivastava S, Lim GS, Tang P, Yang H, et al: SALL4 is a new target in endometrial cancer. Oncogene 34: 63-72, 2015.

30. Kobayashi D, Kuribayashi K, Tanaka M and Watanabe N: Overexpression of SALL4 in lung cancer and its importance in cell proliferation. Oncol Rep 26: 965-970, 2011.

31. Ardalan Khales S, Abbaszadegan MR, Abdollahi A, Raeisossadati R, Tousi MF and Forghanifard MM: SALL4 as a new biomarker for early colorectal cancers. J Cancer Res Clin Oncol 141: 229-235, 2015.

32. Forghanifard MM, Ardalan Khales S, Javdani-Mallak A, Rad A, Farshchian $\mathrm{M}$ and Abbaszadegan MR: Stemness state regulators SALL4 and SOX2 are involved in progression and invasiveness of esophageal squamous cell carcinoma. Med Oncol 31: 922, 2014.

33. Itou J, Matsumoto Y, Yoshikawa K and Toi M: Sal-like 4 (SALL4) suppresses CDH1 expression and maintains cell dispersion in basal-like breast cancer. FEBS Lett 587: 3115-3121, 2013. 